\title{
Avaliação da cobertura vacinal do esquema básico para o primeiro ano de vida*
}

\author{
Assessment of immunization coverage for the basic schedule in children
}

\author{
Alcides S. de Miranda, Ilóite M. Scheibel, Mario R. G. Tavares, Silvia M. P. Takeda \\ Grupo Hospitalar Conceição - Serviço de Saúde Comunitária - Unidade Valáo - \\ Porto Alegre, RS - Brasil
}

\begin{abstract}
Em 1991 avaliou-se a cobertura vacinal em crianças de 12 a 23 meses de idade no território de responsabilidade de um Posto de Atenção Primária à Saúde, na periferla da Zona Norte de Porto Alegre, RS, Brasil, cinco anos após sua implantação, com a finalidade de melhorar a qualidade das açōes de saúde desenvolvidas no serviço. Foram investigadas todas as crianças através de um inquérito domiciliar, observando-se a carteira de vacinas $\theta$ as informaçōes da māe. Em 1986, um inquérito inicial havia identificado uma cobertura vacinal inferlor a $60 \%$ para cada uma das vacinas. A atual cobertura vacinal (doses comprovadas) para trés doses da vacina DPT (Difteria, Pertussis e Tétano), trés doses da Sabin (antipoliomielite), uma dose da anti-sarampo (VAS) e uma dose de BCG săo, respectivamente $87,69,88$ e $79 \%$. Apesar das altas coberturas observadas por tipos de vacinas, quando se verificou para cada criança se o esquema básico do primejro ano de vida estava completo (3 doses de DPT + 3 doses de Sabin +1 dose de VAS + 1 dose de BCG), encontrou-se apenas $75 \%$ das crianças na citada situaçăo. A cobertura vacinal é heterogênea dentro do território, sendo maior naquelas áreas caracterizadas por piores condiçōes socioeconômicas, onde a equipe de saúde havia intensificado esforços. A comparaçāo com o método administrativo de avaliaçāo de cobertura, realizado mensalmente, mostrou a năo-adequaçăo desse, que subestimava a cobertura vacinal. Avaliou-se a situaçăo vacinal das må̀es, para vacina antitetânica, e apenas $49 \%$ das crianças estavam protegidas contra o tétano neonatai. Os dados obtidos subsidlaram a imediata reestruturaçāo das açōes do programa, com vistas a atingir uma cobertura vacinal de $100 \%$, me melhorar a qualidade das aç̌es de saúde prestadas pela equipe.
\end{abstract}

Vacinaçdo, estatística. Esquema de imunizaçao. Avaliaçdo de processos e resultados, cuidados de saúde.

\section{Introduçāo}

Nenhuma ação em saúde é tão efetiva e eficiente quanto vacinar crianças contra as principais doenças preveníveis por imunização. Entretanto, ainda existe um hiato entre o potencial dessa prática e suas reais contribuiçōes para a sobrevivência infanti1 $^{14.16 .21,22}$. Anualmente morrem cerca de três milhōes de crianças e outras tantas tornam-se inválidas devido a essas doenças ${ }^{14,22}$. A cobertura vacinal - definida como o percentual de uma populaçăo-alvo que foi vacinada ${ }^{19,35}$ - nos chamados "paŕses em desenvolvimento" ainda está aquém da esperada $a^{21,39}$. Para atingir uma cobertura vacinal adequada $€$ preciso fundamentalmente ações insti- tucionais conjugadas e organizadas pelo setor público em diversos níveis ${ }^{15}$.

Em esforço conjunto a Organizaçāo Mundial da Saúde (OMS), a Organização Panamericana de Saúde (OPS) e os governos da América Latina e Caribe desenvolveram o Programa Ampliado de Imunizaçōes (PAI) ${ }^{37}$, definindo metas e diretrizes para a superação da defasagem entre esse potencial e seu desempenho.

No Brasil, o Programa Nacional de Imunizaçðes (PNI) foi introduzido em $1973 \mathrm{com}$ o objetivo de controlar ou erradicar a poliomielite, 0 sarampo, a difteria, o tétano, a coqueluche e a tuberculose, mediante imunização sistemática, com a meta de atingir $100 \%$ de cobertura vacinal

* Pesquisa subvencionada pela Fundacão de Amparo d̀ Pesquisa (FAPERGS), Processo n $n^{\circ}$ 90.02605-0. Trabalho apresentado no IV Congresso Brasileiro de Medicina Geral Comunitária, Porto Alegre, RS, 1991; e IV Congresso Internacional de Medicina de Familia, Montevidéti-Uruguai, 1993.

Separatas/Reprints: Mario R.G. Tavares - Av. Joāo XXIII, 253/I22 - Bl. 6 - São Sebastiăo - 91060-100 - Porto Alegre, RS - Brasil Recebido em 27.4.1994. Reopresentado em 15.2.1995. Aprovado em 16.3.1995. 
para o primeiro ano de vida ${ }^{20}$. Apesar desses esforços, em 1988 a cobertura vacinal no país ainda era aquém da expectativa: $63 \%$ para a antisarampo (VAS), 62\% para a tríplice (DPT) e Sabin (antipoliomielite), e $61 \%$ para a BCG (antituber-

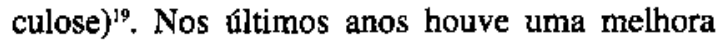
sensivel. Em 1991 as estatísticas oficiais noticiam taxas de cobertura de $97 \%, 89 \%, 76 \%$ e $99 \%$, respectivamente ${ }^{18}$.

A analise das taxas de cobertura por regiōes do Brasil revela que a regiāo Sul apresenta relativamente maior cobertura para todas as vacinas ${ }^{11,34,37,41}$.

Em 1986, foi instalado um posto de saúde na periferia da Zona Norte de Porto Alegre, RS. Na ocasiāo, realizou-se um inquérito com amostra sistemática de $20 \%$ dos domicilios, que demonstrou uma cobertura de $53 \%$ para a Sabin, $50 \%$ para a DPT e $56 \%$ para a VAS, entre as crianças de 12 a 23 meses de idade ${ }^{42}$.

A saúde da criança foi eleita como prioridade e se introduziu um conjunto de açôes programáticas com o objetivo de impactar favoravelmente a saúde desse grupo etário. A vacinação constitui-se numa das açōes primordiais do programa, compreendendo atividades de treinamento e motivação da equipe para identificar crianças não vacinadas e vaciná-las, atividades comunitárias para sensibilizar a população da relevância do problema, vacinação casa a casa nas épocas de campanhas govemamentais, vacinaçăo sistemática de usuários, busca ativa de casos, avaliação mensal da cobertura vacinal por método administrativo (obtida com base no número de crianças vacinadas no mês e no número estimado de crianças na comunidade) e busca domiciliar a faltosos. As vacinas realizadas naquela época não incluíam BCG, que passou a ser feita no Estado do Rio Grande do Sul em menores de 7 anos, a partir de 1990. Conjuntamente coordenou-se um programa de assistência ao pré-natal, incluindo imunização contra o tétano neonatal.

Em 1991, realizou-se novo inquérito domiciliar, na mesma populaçāo, para reavaliar a cobertura vacinal após 5 anos da implantação do posto e validar a cobertura vacinal encontrada com aquela obtida pelo método administrativo. Para inferir a qualidade da atençāo dispensada foi comparada a cobertura local com as obtidas no município e no Estado do Rio Grande do Sul. Ainda, avaliou-se a cobertura vacinal contra o tétano neonatal.

Os resultados obtidos no presente trabalho deverão subsidiar a redefinição das táticas, com vistas a atingir a meta de cobertura vacinal de $100 \%$.

\section{Material e Método}

O presente estudo foi realizado em uma das 13 unidades avançadas de um serviço de Atenção Primária à Saúde, localizado na periferia da zona Norte de Porto Alegre, RS. As equipes de saúde, de composiçăo multiprofissional, sāo responsáveis por territórios-área abrangendo populações que variam de 3 a 20 mil pessoas. A área de abrangência da referida unidade compreende cerca de 7,5 mil habitantes.

O método estatístico de obtenção da cobertura, baseado em inquérito domiciliar, foi escolhido por ser mais adequado às áreas de difícil caracterização da população-alvo, por contemplar as coberturas vacinais das populações que migram, pela possibilidade de eliminar-se sub-registros e duplicidade de dados e, ainda, por permitir vacinar as crianças em atraso identificadas durante o estu$\mathrm{do}^{5,8,11,22,23,28,36}$.

Todos os domicílios da área foram visitados e todas as crianças de 12 a 23 meses de idade foram identificadas. Optou-se por esse grupo que permitia avaliar crianças que já deveriam estar com seu esquema básico de vacinação para o primeiro ano de vida completo ${ }^{3.9}$. Para cada criança identificada foi aplicado um questionário padronizado à mãe ou a quem cuidava da criança na maior parte do tempo (responsável).

As doses de vacinas foram classificadas na categoria confirmadas quando comprovadas no cartão de vacinas/cartão da criança pelo carimbo do posto de vacinação, data e a rubrica do funcionário, e no caso da BCG, também pela inspeção da cicatriz característica na região deltóide direita. Nos casos em que o cartão não estava disponível ou quando nåo havia registro nesse, mas o responsável era capaz de descrever como a vacina foi aplicada, classificaram-se como doses nâo confirmadas ${ }^{3}$. Foi considerada como adequadamente vacinada toda criança com pelo menos três doses de Sabin, três doses de DPT, uma dose de VAS e uma de $\mathrm{BCG}^{10,19,33,40,43,45}$.

As outras informaçōes coletadas foram: gênero e data de nascimento da criança, endereço, posto de vacinação em que foi efetuada a última vacina, vacinação antitetânica da mãe na época do nascimento da criança e escolaridade do responsável. Esta última foi a variável selecionada para análise socioeconômica ${ }^{3.26,47}$. Uma das crianças, inicialmente eleita, por não se conseguir determinar com segurança a data de seu nascimento, foi excluída do estudo $(0,9 \%)$. 
Para análise da cobertura vacinal foram apresentados dois percentuais: doses confirmadas e doses confirmadas acrescidas das doses não confirmadas. A verdadeira cobertura vacinal é provavelmente algum valor intermediário entre um e outro percentual ${ }^{3}$. Por exemplo, na Figura 1, a cobertura vacinal para tríplice é algum valor entre $81 \%$ (doses confirmadas) e $94 \%$ (doses confirmadas e nāo confirmadas). Para comparaçåo temporal foram usadas as doses confirmadas, pois o estudo de 1986 só considerou essas. Para comparação com método administrativo e comparaçăo espacial (com Porto Alegre e com o Estado do Rio Grande do Sul) utilizaram-se as doses confirmadas mais metade das doses não confirmadas, buscando-se diminuir o tamanho do erro sistemático.

Considerou-se protegida contra o tétano neonatal a criança cuja mãe recebera pelo menos duas doses de vacina antitetânica, sendo a última há menos de 5 anos do nascimento da criança, segundo critério da $\mathrm{OMS}^{[, 2,8,12,24,27,44}$.

$O$ teste estatístico utilizado para comparar coberturas foi o Teste $Z$. Para investigar associaçāo entre cobertura vacinal da criança e alfabetização da mãe foi utilizado o Teste Exato de Fisher. Para verificar associação entre níveis de escolaridade do responsável e esquema básico de vacinação completo para o primeiro ano de vida utilizou-se o qui-quadrado ${ }^{30}$.

\section{Resultados}

Visitaram-se os 1.599 domićlios ocupados, identificando-se 108 crianças entre 12 e 23 meses de idade, e o cartão de vacinas/cartão da criança foi inspecionado em $82 \%$ delas. Somente em um caso o responsável referiu não ter cartāo, e em $17 \%$ o cartāo não estava disponível, utilizando-se então somente a informaçāo referida pelo responsável.

\section{Cobertura Vacinal para cada Tipo de Vacina e Esquema Básico}

As coberturas vacinais para as terceiras doses das vacinas DPT e Sabin, uma dose da VAS, uma dose de BCG e esquema básico encontram-se na Figura 1. A maior cobertura encontrada foi para a vacina Sabin, que utilizando as doses nåo confirmadas alcança $97 \%$. A cobertura para VAS foi superior à esperada, 94\%, enquanto a vacina BCG foi a mais baixa, com $84 \%$.

Apesar das altas coberturas observadas por

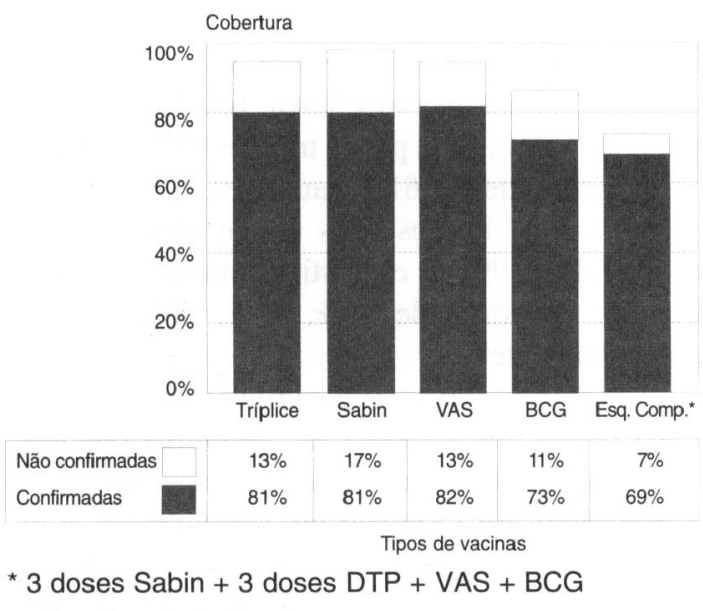

Flgura 1 - Cobertura vacinal do esquema básico para 0 primeiro ano de vida em crianças de 12-23 meses, segundo tipos de vacinas, 1991.

tipo de vacina, quando se verificou para cada criança se o esquema básico do primeiro ano de vida estava completo, encontrou-se uma taxa menor: apenas $75 \%$.

\section{Cobertura Vacinal 5 Anos após a Implantação do Serviço e Diferentes Métodos de Avaliação da Cobertura}

A atual cobertura vacinal considerando somente doses confirmadas quando comparada a de 1986, mostrou um aumento de 14 pontos percentuais para Sabin, 18 para DPT e 21 para VAS (Fig. 2). As diferenças verificadas entre as coberturas nos dois períodos são estatisticamente significativas $(p<0,01)$.

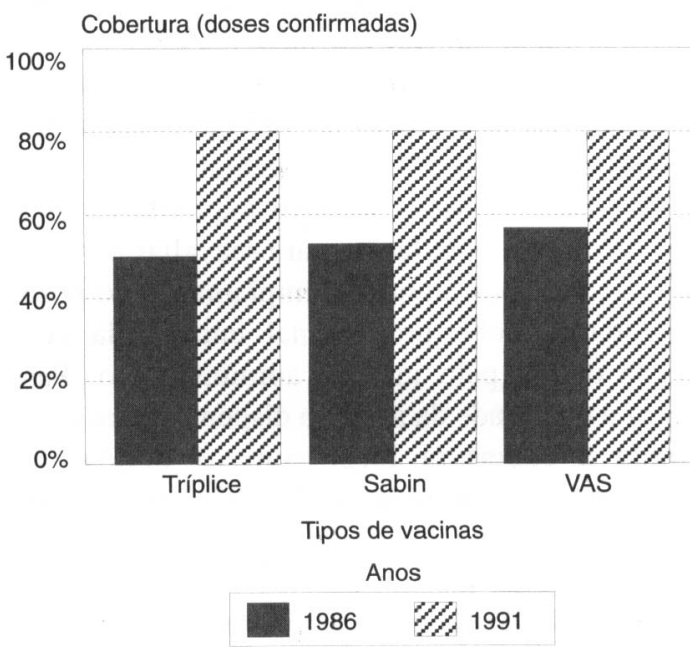

Figura 2 - Coberturas vaclnals para o primeiro ano de vida, segundo tipos de vacinas anos (1986 e 1991). 


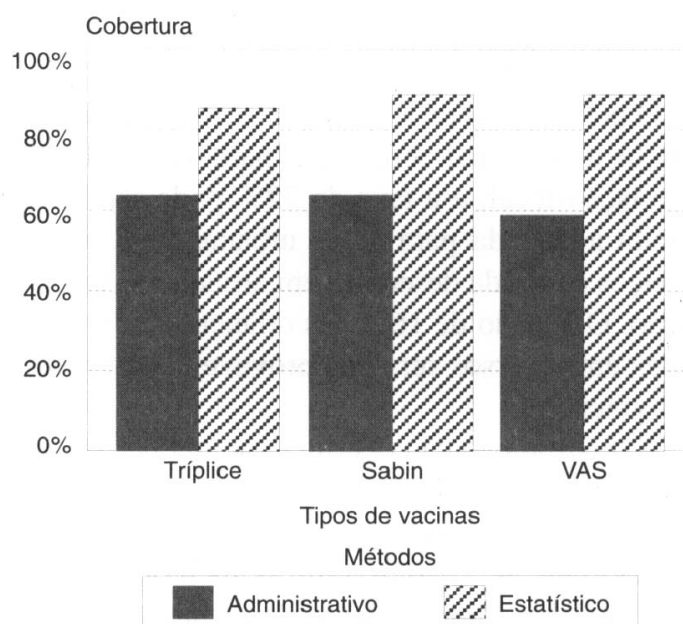

Figura 3 - Cobertura vacinal segundo tipos de vacinas e métodos administrativo e estatístico, 1991.

A cobertura vacinal obtida pelo método estatístico foi comparada àquela obtida pelo método admis. trativo (Fig. 3). O motivo da menor cobertura demonstrada pelo método administrativo deve-se principalmente ao denominador baseado no inquérito de 1986, mas que no momento superestimava em 2060 número de crianças da faixa etária estudada.

Para avaliar a utilizaçāo do serviço verificou-se o local onde foi feita a última vacina recebida pela criança. Cerca de $72 \%$ das crianças utilizaram o posto de saúde estudado, enquanto $12 \%$ vacinaram em outros dois locais próximos. Embora $16 \%$ das crianças tenham sido vacinadas em vários outros locais, nenhum desșes foi responsável pela vacinaçāo de mais de duas crianças.

\section{Cobertura Vacinal por Setores Censitários}

Quando foi analisada a cobertura vacinal para o esquema básico completo por setores censitários da Fundaçăo Instituto Brasileiro de Geografia e Estatística (IBGE), observa-se que não há homo-

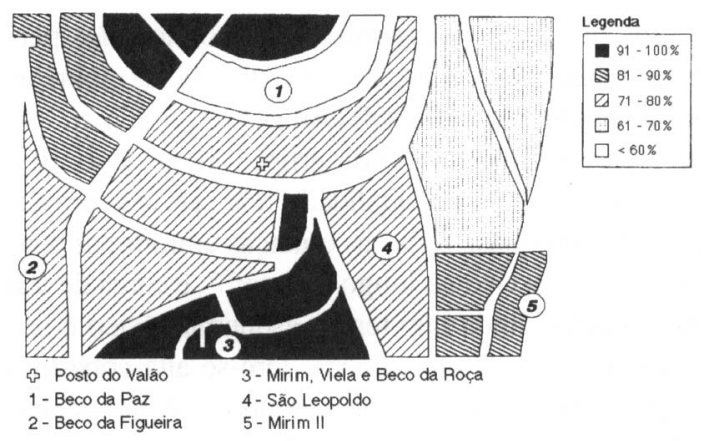

Figura 4 - Cobertura vacinal para o esquema básico do primeiro ano de vida por setores censitários do IBGE, 1991. geneidade (Fig. 4). Alguns setores alcançam 100\% ou próximo disso, outros nāo chegam a $60 \%$. Alguns setores, com baixas coberturas, estāo localizadas próximos ao posto de saúde estudado. Outros, cuja população encontra-se em piores condiç̧es socioeconômicas, apresentaram as melhores coberturas, como as micro-áreas (Fig. 4).

\section{Cobertura Vacinal do Posto de Saúde em Porto Alegre e no Estado do Rio Grande do Sul}

A cobertura vacinal para o Município de Porto Alegre, de janeiro a outubro de 1991, atingiu $71 \%$ para DPT e Sabin, 69\% para VAS e $79 \%$ para BCG. Quando comparada às taxas acima, a cobertura vacinal no posto de saúde foi maior para todos os tipos de vacina, exceto BCG. As diferenças foram significativas para Sabin, DPT e VAS, mas não para $\mathrm{BCG}(\mathrm{p}<0,01)$.

No Estado do Rio Grande do Sul, a cobertura vacinal no mesmo período, registra $82 \%$ para DPT e Sabin, $83 \%$ para VAS e $85 \%$ para BCG. Novamente no Posto de Saúde as coberturas foram maiores para todos os tipos de vacina, exceto BCG. Entretanto, não houve diferenças estatisticamente significativas entre essas coberturas.

\section{Cobertura Vacinal da Criança e Escolaridade Materna}

Cerca de 70\% das mães completaram mais de 4 anos de escola formal. Do total, $6 \%$ eram analfabetas, situação semelhante àquela encontrada em $1986^{42}$. Não se demonstrou associaçāo entre escolaridade materna e cobertura vacinal da criança.

\section{Cobertura Vacinal Antitetânica da Mảe}

Em $15 \%$ dos casos não houve informaçăo sobre a situação vacinal antitetânica da mãe na

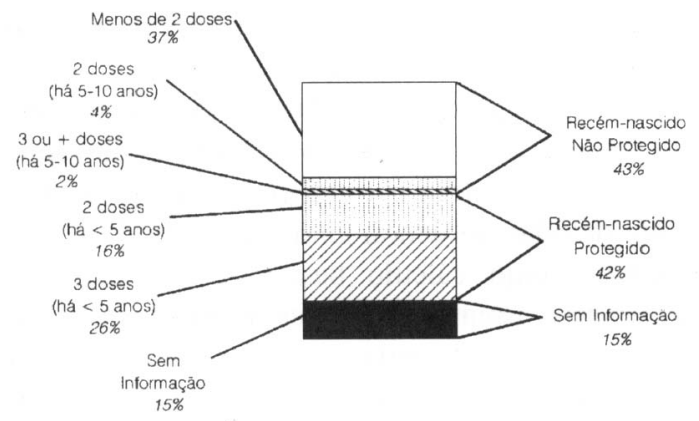

Figura 5 - Estado vacinal da māe para antitetânica e proteçăo para tétano neonatal, 1991. 
ocasião do nascimento da criança. Na maioria das vezes a própria māe não soube informar seu estado vacinal. Em alguns poucos casos de crianças adotivas, abandonadas ou ófăs năo havia registro disponivel. Entre aquelas que informaram, observou-se que $49 \%$ das crianças estavam protegidas contra o tétano neonatal (Fig. 5).

\section{Discussão}

A cobertura vacinal é um importante indicador de saúde das populações e da qualidade da atenção dispensada pelos serviços ${ }^{27}$. $\mathrm{O}$ estudo desse indicador, além de apontar aspectos da saúde infantil e da atuação dos serviços, subsidia o processo de planejamento, especialmente a reestruturação das açōes.

Avaliar é uma prática pouco comum. $O$ presente estudo evidencia a possibilidade de realizar avaliaçōes com relativamente poucos recursos, curto período de tempo, metodologia simples, e resultados de aplicabilidade imediata.

A atual cobertura vacinal na área de abrangência do posto estudado pode ser considerada boa quando realizadas comparaçōes temporal (1986) e espacial (Munícipio e Estado), evidenciando-se significativa melhora. Embora o estudo nāo tenha sido concebido para avaliar causas de modificações da cobertura vacinal nos dois momentos, as atividades realizadas com a implantação do programa de vacinação, a utilização do posto como principal referência e a baixa escolaridade materna mantida nos permitem supor que a melhor cobertura seja atribuível, ao menos em parte, à atuação da equipe de saúde.

Por outro lado, $25 \%$ das crianças estudadas não tinham o esquema básico de vacinação completo, o que nos faz repensar a abordagem das crianças que entram em contato com o serviço em qualquer ocasiāo e por qualquer motivo (oportunidades perdidas) ${ }^{8.9 .17 .45}$. Além disso, reforça a necessidade de treinamento periódico das equipes, que costumam superestimar contra-indicaçбes".

As diferenças identificadas nas taxas de cobertura vacinal, entre as duas metodologias de avaliação (administrativa e estatística), são principalmente devidas ao denominador do método administrativo, cujo cálculo baseia-se em taxas de crescimento irreais. Parte dessa diferença ainda pode ser explicada pelas crianças vacinadas em outros serviços (12\%), que o método administrativo não considera. Faz-se necessário o redimensionamento perídico da populaçåo-alvo, o que adequará a estimativa da cobertura vacinal através do método administrativo.

O posto de saúde situado no território estudado é hoje a principal referência para vacinaçāo da populaçāo-local, e a taxa de $72 \%$ pode ser considerada adequada para áreas urbanas onde vários serviços de saúde desenvolvem atividades de vacinação. $O$ achado de $16 \%$ das crianças serem vacinadas em diversos serviços provavelmente reflete a migração.

A distribuição da cobertura vacinal por setores censitários é heterogênea, o que não se explica pela distância em relação ao posto de saúde. Os setores onde se encontraram as melhores coberturas vacinais, independente da distância, correspondem àqueles cuja população possui renda familiar mais baixa ${ }^{42}$, onde a equipe de saúde havia intensificado sua atuaçāo desde 1986. Esse achado acrescenta mais uma evidencia da efetividade da abordagem por micro-áreas ${ }^{20,47}$, e nesse momento a equipe deve priorizar as áreas de menor cobertura, sem descuidar das demais.

É conhecida a associação entre escolaridade materna e situaçåo de saúde infantil ${ }^{3,4,26,32}$. Analogamente, espera-se que melhores coberturas vacinais estejam associadas com melhor escolaridade materna. Tal associação não foi identificada, o que pode ser justificado pela pequena base populacional estudada, ou por fatores confundindo a associaçāo, tais como a atuaçảo concentrada da equipe de saúde nas micro-áreas de maior risco.

Sobre a cobertura para o tétano neonatal, o percentual encontrado de $49 \%$ pode ser considerado bom quando comparado a outros locais ${ }^{1,2,12,25}$. Entretanto, se levarmos em conta que aproximadamente $90 \%$ das gestantes da área fazem pré-natal no posto estudado do Valão ${ }^{46}$, percebem-se deficiências na atuação da equipe.

\section{Comentários}

Os resultados e conclusões proporcionados permitiram dar início a um processo de reflexão sobre as ações e métodos desenvolvidos pela equipe com vistas a elevar a motivação do grupo. Além disso, permitiram questionar a qualidade dos serviços prestados, quando quase todas as crianças entram em contato com o serviço ao longo do ano ${ }^{17,31}$, e cerca de um quarto delas ainda não tem o esquema básico completo, quando paradoxalmente tem-se alta cobertura de pré-natal e baixa proteção contra o tétano neonatal. Diversos treinamentos e oficinas tiveram início com o objetivo de qualificar as ações da equipe. 
O processo de avaliação, com o sentido de auditar o serviço, motivou a equipe a avaliar suas açőes de maneira mais global. Logo após o término desse estudo, iniciaram-se mais três projetos de avaliaçāo do trabalho após cinco anos do início das atividades: modificaçăo das condiçžes de saúde da população, qualidade da atenção prénatal e qualidade da atenção à criança.

$O$ desenvolvimento do presente projeto, relativamente simples, rápido, objetivo, de baixo custo operacional, alta factibilidade e ético, proporcionou resultados concretos e imediatos para equipe $e$ população envolvidas, capaz de estimular outros projetos de pesquisa.

Por outro lado, os resultados obtidos reafirmam a necessidade de incrementar ações preventivas básicas. Há atividades a serem implementadas por equipes de saúde, relativamente simples, cultural- mente incorporadas, de baixo custo e alto impacto que não permitem aceitar resultados inferiores a $100 \%$ da meta estabelecida. A vacinação a crianças e gestantes são prototipos dessas ações. A par da atuação estratégica, holística e participativa tão discursada e poucas vezes realizada ${ }^{13}$, é uma lástima que os técnicos da Atenção Primária não cumpriam o direito de vacinar $100 \%$ das crianças ${ }^{6,7,38}$ sob a responsabilidade compartilhada pelos pais e pelo Setor Saúde.

\section{Agradecimentos}

Aos Professores Fernando Barros e César Victora, da Universidade Federal de Pelotas, RS, pela valiosa colaboração. Aos revisores que avaliaram o manuscrito, pelas valiosas críticas e sugestðes.

\section{Referências Blbliográflcas}

1. AMSTEY, M. S. et al. Neonatal passive immunization by maternal vaccination. Obstet. Gynecol., 63: 105-9, 1984.

2. ARNOLD, R. et al. Mortality from neonatal tetanus in Indonesia: results of two surveys. Bull. World Health Organ., 64: 259-62, 1986.

3. BARROS, F, C. \& VICTORA C. G. Análise dos dados. In: Barros, F. C. \& Victora C. G. Epidemiologia da saúde infantil: um manual para diagnósticos comunitários. São Paulo, Hucitec/Unicef, 1991. p. 137-50.

4. BARROS, F. C. \& VICTORA C. G. Indicadores socioeconômicos, ambientais e demográficos. In: Barros, F. C. \& Victora C. G. Epidemiologia da saude infantil: um manual para diagnosticos comunitátos. São Paulo, Hucitec/Unicef, 1991. p. 42-52.

5. BEGG, N. et al. COVER (cover of vaccination evaluated rapidly): description of the England and Wales scheme. Public Healtb., 103: 81-9, 1989.

6. BRASIL. Leis, Decretos, etc. Constituigâo federal de 1988: Artigo 227, Capitulo VII. Brasília, Congresso Nacional, 1988.

7. BRASIL. Leis, Decretos, etc. Estatuto da crianca $e$ do adolescente: Let 8069 de 13/07/90 Brasília, Congresso Nacional, 1990.

8. CENTERS FOR DISEASE CONTROL, Immunization Practices Advisory Committee. General recommendations on immunization: guidelines from the Immunization Practices Advisory Committee. JAMA, 262: 187-91, 1989.

9. CLEMENTS, C. J. et al. 1988 New Zealand national immunization survey; methodology. N. Z. Med. J., 102: 320-2, 1989.

10. COLVER, A. Immunization and general practice. Lancet, 1: 906, 1989.

11. COSNER, A. M. et al. Cobertura e motivos de atrasos vacinais em crianças de vila periférica da Grande Porto Alegre. Rev. AMRIGS, 32: 58, 1988

12. DIXON, A. M. \& BIBBY, J. A. Tetanus immunization state in a general practice population. Br. Med. J, 297: 598, 1988.

13. DUNCAN, B.B. et al. A importância das medidas preventivas. In: Duncan, B. B. et al. Medicina ambulatorial: condutas clinicas em atenção primária. Porto Alegre, Ed. Artes Médicas, 1990. p. $169-71$

14. EKANEM, E. E. A ten year review of morbidity from childhood preventable diseases in Nigeria: how successful is the expanded programme of immunization (EPI)? J. Trop. Pediat., 34: 323-8, 1988.

15. EXPANDED PROGRAMME ON IMMUNIZATION. Programme evaluation. Whly. Epidemiol. Rec., 56: $276-8,1981$.

16. FOEGE, W. H. Prospects for universal immunization: strategics for achievement, Rev. Infect. Dis., 11 (Suppl. 3); S659-62, 1989.

17. FOSTER, S. O. Immunization opportunities taken and missed. Rev. Infect. Dis, 11 (Suppl. 3): $\$ 629-30,1989$.

18. FUNDAÇÃO IBGE. Anuârio estatístico do Brasil. Rio de Janeiro, IBGE, 1993.

19. FUNDAÇÃO NACIONAL DE SAÚDE. Manual de normas de vacinação. Brasília, DF, Departamento de Operaçōes, 1994.

20. FUNDACÄO NACIONAL DE SAÚDE. Manual de procedimento para vacinação. Brasília, DF, 1993.

21. HENDERSON, R, H. et al. Immunizing the children of the world: progress and prospects. Bull. World Health Ongan., 66:535-43, 1988.

22. HENDERSON, R. H. \& KEJA, J. Global control of vaccine preventable diseases: how progress 
can be evaluated. Rev: Infect Dis, 11 (Suppl. 3): $\$ 649-54,1989$.

23. HENDERSON, R. H. \& SLNDARESANi, T. Clusters sampling to assess immunization coverage: a review of experience with simplified sample method. Bull. Wonld Health Organ., 60: 253-60, 1982.

24. HOWIE. J. G. R. Anyone for tetanus? [Editorial]. Br. Med. J., 297:570-1, 1988.

25. INSEL, R. Maternal immunization to prevent neonatal infections [Editorial]. A. Engl. I. Med. 319: 1219-20, 1988.

26. JARMAN, B. Social and economic factors in ill health. In: Jarman. B. Primary care. Oxford. Heinemann Medical, 1988. p. 31-58.

27. JARMA 5 . B. et al. Lptake of immunization in discrict health authorities in England. $B r$. Med. J., 296: 1775-8, 1988.

28. JOSEPH, A. et al. Por una mejor cobertura de la immunización. Foro Mundial Salud, 9: 342-6. 1988.

29. KADT, E. \& TASCA, R. Enfrentando a iniqüidade: estratégias de intervenção que enfocam chances de saúde. In: Kadt, E. \& Tasca. R. Promovendo a eqüidade: um novo enfoque com base no setor da satide. Sào Paulo. Hucitec, 1993. p. 31-43.

30. KIRKWOOD, R. B. Essentials of medical statistics. London, Ed. Blackwell Scientific, 1988.

31. KOFOED, $P$. L. et al. Immunization in a curative setting. Br. Med.J, 301: 593-4, 1990

32. MENDES, E. V. Lima agenda para a saúde no Brasil. Brasília, 1993. [Documento apresentado no painel de debate: A Crise da Saúde no Brasil: Impasses e Perspectivas. Fundaçào do Desenvolvimento Administrativo - FLXDAP. 2 de julho de 1993, São Paulo, SP\}.

33. MIDDLETON, D. B. Immunization. Prim. Care. 17: 713-30, 1990 .

34. MINISTÉRIO DA SAĹtDE. Programa Nacional de Imunizaçōes. A criança, a tacina e o munict pio. Brasília, DF. Ed. OCB, 1993

35. MINISTÉRIO DA SAÚDE. Manual de vacinação. Brasília. DF, Centro de Documentação, 1984. Série A: Normas e manuais técnicos, 15.
36. MINISTÉRIO DA SAĹDE. Manual para campanhas de tacinaçâo. Brasilia. DF, Centro de Documentaçào, 1988. (Série A: Normas e manuais técnicos, 41).

37. MINISTÉRIO DA SAĹDE. Programa ampliado de imunizaçoes (PAI). Brasilia, DF, Centro de Documentaçào. 1988.

38. ORGANIZACÁO DAS NACÕES LNIDAS, Declaração mundial sobre a sobretitência, a proteça e o desentolitimento da criança nos anos 90 e plano de açäo para a stia imple. mentaçāo. Nova Iorque. 1990.

39. ORGANIZACION PANAMERICANA DE LA SALLD. Las condiciones de salud en las Américas. Washington, D. C. . 1990. (OPS Publicación cientifica $n^{\circ} 524$ ).

40. RAMALINGASWAMI. V. Importance of vaccines in child sunival. Ret. Infect. Dis.. 11 (Suppl. 3): $5498-502,1989$.

41. SECRETARIA DA SAL̈DE E DO MEIO AMBIENTE - RS. Vigilância sanităria. Bol. Epidemiol. (4): 1-4., 1988 .

42. SERVIÇO DE SAĹDE COMLNITÁRIA. Equipe de Epidemiologia. Grupo Hospitalar Conceiçào. Diagnóstico da comunidade do Valào. Porto Alegre, 1986.

43. SLEIGHT. G. Lptake on immunization [Letter] Br. Med.J., 297: 626, 1988.

44. SIMONSEN, O. et al. Immunity against tetanus and effect of revaccination $25-30$ years after primary vaccination. Lancet, 2: 1240-2. 1984.

45. SOKHEY. J. et al. The expanded programme on immunization: a decade of progress in India. Ann. Trop. Paediatr. 9: 24-9, 1989.

46. TAKEDA, S. M. P. Atraliaçào de serriço de atençào primária: modificaçòes nos indicadores de saúde e qualidade de atenção. Pelotas, 1993. [Dissertaçào de Mestrado. Lniversidade Federal de Peloras/RS].

47. LNGLERT, C. V. S. Territorialização em sistemas de saúde. In: Mendes, E. V. et al. Distrito sanitärio: o processo social de mudança das práticas sanitarias do sistema unico de saíde. Sào Paulo, Hucitec, 1993. p. 221-35.

\begin{abstract}
Immunization coverage was evaiuated in all 12-23 month-old children living in the area were five years before a Primary Care Practice had been set up. All children were investigated through home visits, checking of the immunization chart and relying on mothers ${ }^{\prime}$ information. In 1986, a baseline study had identified an immunization coverage of under $60 \%$ for each of the scheduled vaccines. The current study confirmed that coverage was of $87 \%$ for three doses of DTP, $89 \%$ for Sabin, $88 \%$ for one dose of measles vaccine and $79 \%$ for BCG. Despite the high coverage achieved for each specific vaccine, when the basic schedule for the first year was verified, it was observed that only $75 \%$ of the children had received the full scheme. Immunization coverage is uneven in different census tracts, being higher in the poorest and more remot areas, where seam the health has given extra attention. A comparison with the routine administrative evaluation of the immunization coverage showed that this underestimated the real coverage. Maternal immunization uptake was also evaluated (antitetanus vaccine during pregnancy) and only $49 \%$ of the women were found to be adequately protected. The information collected led to a reorganization of the whole immunization program in a $100 \%$ coverage.
\end{abstract}

Vaccination, statistical. Immunization schedule. Outcome and process assessment, health care. 